\title{
La competencia transversal de comunicación efectiva en estudios de máster en el ámbito de la ingeniería civil y la construcción
}

Víctor Yepes ${ }^{a}$ y José V. Martíb

aCITECH. Equipo de Innovación y Calidad Educativa EXCELCON. Departamento de Ingeniería de la Construcción y Proyectos de Ingeniería Civil. Universitat Politècnica de València. vyepesp@cst.upv.es y ${ }^{b}$ ICITECH. Equipo de Innovación y Calidad Educativa EXCELCON. Departamento de Ingeniería de la Construcción y Proyectos de Ingeniería Civil. Universitat Politècnica de València.jvmartia@cst.upv.es

\begin{abstract}
This paper presents the results of a perception study of the M.Sc. degree in concrete engineering and construction management students in relation to the learning outcomes for effective communication crossdisciplinar competence. An anonymous questionnaire using a Likert scale of 12 questions was used. Principal components analysis was used to identify underlying latent variables that explain the pattern of correlations. We have identified three principal components: skills related to oral communication, competence related to the organization and clarity of ideas and expertise related to the use of English. It has also proposed a multiple regression model to explain the variable "communicate clearly and effectively in an oral presentation, using the strategies and appropriate means, depending on the audience and objectives." The results allowed the design based on active learning methods and activities in order to assess this crossdisciplinar competence activities. Changing a written scientific paper into an oral presentation and writing a scientifica paper were the activities. The oral presentation of a scientific paper and writing a scientific article. The group work and the case study were the methodologies used.
\end{abstract}

Keywords: crossdisciplinar competences, effective communication, civil engineering, M.Sc. degree, multivariate analysis. 
La competencia transversal de comunicación efectiva en estudios de máster en el ámbito de la ingeniería civil y la construcción

\begin{abstract}
Resumen
La comunicación presenta los resultados de la percepción que realizan alumnos de los másteres de ingeniería del hormigón y de planificación y gestión en la ingeniería civil en relación con los resultados de aprendizaje de la competencia transversal comunicación efectiva. Se ha realizado para ello una encuesta anónima utilizando una escala Likert de 12 preguntas. Se ha elaborado un análisis factorial mediante el método de componentes principales para identificar las variables subyacentes o factores que expliquen la configuración de las correlaciones. Se han reconocido tres componentes principales: competencias relacionadas con la comunicación oral, competencia relacionada con la organización y claridad de las ideas y competencia relacionada con el uso del idioma inglés. Además, se ha propuesto un modelo de regresión múltiple para explicar la variable "comunicarse de forma clara y eficaz en una presentación oral, utilizando las estrategias y los medios adecuados, en función del público y los objetivos". Los resultados han permitido el diseño de actividades basadas en metodologías activas para la evaluación de esta competencia transversal. Se trata de la presentación oral de una comunicación científica y la redacción de un artículo científico. Las metodologías a emplear serán las de trabajo en grupo y el estudio de caso.
\end{abstract}

Palabras clave: competencias transversales, comunicación efectiva, ingeniería civil, máster, análisis multivariante.

\title{
1. Introducción
}

La evaluación de las competencias transversales se ha convertido en un objetivo estratégico de la Universitat Politècnica de València (UPV), impulsado por el Vicerrectorado de Estudios, Calidad y Acreditación. Para el estudiante resulta muy importante adquirir dichas competencias y acreditar su formación en competencias, y para el empleador y la sociedad, resulta muy relevante conocer el nivel adquirido por el egresado. Para la UPV supone una mejora continua de sus títulos universitarios, con un valor añadido innegable, facilitándose de esta forma la acreditación nacional e internacional de sus títulos.

En el ámbito de la ingeniería civil y la construcción, el Máster Universitario en Ingeniería del Hormigón (MUIH) y el Máster Universitario en Planificación y Gestión en Ingeniería Civil (MAPGIC) son dos títulos oficiales verificados (Pellicer et al., 2013; 2014; Yepes et al., 2012). Además, el MUIH cuenta con la acreditación EUR-ACE® (http://www.enaee.eu/eur-ace-system). 
Con el objeto de evaluar la competencia transversal "comunicación efectiva", se han elegido las asignaturas "Modelos predictivos y de optimización de estructuras de hormigón” del MIUH y "Gestión de la Innovación en el Sector de la Construcción”, del MAPGIC, de las cuales el primer autor es profesor responsable.

\section{Objetivos}

Los objetivos de la comunicación son los siguientes:

1. Valoración de la percepción de los alumnos de posgrado en el ámbito de la ingeniería civil de la importancia de los resultados de aprendizaje de la competencia transversal "comunicación efectiva".

2. Conocer los factores subyacentes en los resultados de aprendizaje con el propósito de orientar la evaluación de dichos resultados de aprendizaje.

3. Elaborar un modelo explicativo basado en una regresión múltiple de la variable "Comunicarse de forma clara y eficaz en una presentación oral, utilizando las estrategias y los medios adecuados, en función del público y los objetivos".

4. Diseño de actividades y evaluación de los resultados de aprendizaje a la vista de los resultados de la encuesta realizada, basándose en el uso de metodologías activas.

\section{Desarrollo de la innovación}

La innovación planteada consiste en fundamentar el diseño de actividades basadas en metodologías activas de forma que se permita la evaluación de los resultados de aprendizaje más importantes de la competencia transversal "comunicación efectiva". La novedad consiste en haber realizado, previo al diseño de las actividades, una labor de recogida de datos formal a través de una encuesta que permita valorar la percepción de los alumnos respecto a los resultados de aprendizaje "a priori". Estos resultados de aprendizaje, atendiendo al nivel de estudios de máster, son los detallados en la página web de la UPV: http://competencias.webs.upv.es/wp/

Los resultados obtenidos de la encuesta ha permitido comprobar que existen tres componentes subyacentes a la competencia de comunicación efectiva, que son los siguientes: competencia relacionada con la comunicación oral, competencia relacionada con la organización y claridad de las ideas y competencia relacionada con el dominio del idioma inglés.

Por tanto, atendiendo a dicha encuesta, se proponen dos actividades basadas en metodologías activas para la evaluación de la competencia transversal: 
La competencia transversal de comunicación efectiva en estudios de máster en el ámbito de la ingeniería civil y la construcción

- Presentación oral: Por una parte los alumnos, en grupos de 3, deberán leer un artículo científico en inglés y exponer, en un máximo de 15 minutos, el contenido del mismo al resto de la clase. El profesor proveerá a los alumnos de una rúbrica que servirá para evaluar la presentación. La presentación será evaluada tanto por el profesor como por el resto de los alumnos, con lo cual se refuerza el aprendizaje de la competencia: http://excelcon.blogs.upv.es/2015/02/06/como-podemosevaluar-una-presentacion-oral/

- Redacción de un artículo científico: El profesor utiliza la metodología de estudio de caso para evaluar un artículo científico tipo. http://excelcon.blogs.upv.es/2013/05/20/estudio-de-caso-lectura-y-comprensionde-un-articulo-cientifico/ Posteriormente, se explica la estructura de un artículo y se les da una plantilla para evaluar un artículo tipo: http://excelcon.blogs.upv.es/2014/03/10/tienen-nuestros-alumnos-que-aprender-aescribir-articulos-cientificos/ Una vez han adquirido dicho conocimiento, los alumnos, en grupos de 3 , desarrollan un trabajo con resultados al que deben dar la estructura de artículo científico. En el caso del MIUH, se trata de la optimización de un puente losa utilizando la metodología de la superficie de respuesta.

Se puede comprobar que, con las dos actividades propuestas y las metodologías activas planteadas, se cubren las tres componentes principales detectadas en este estudio.

\section{Resultados}

Para conocer la percepción de los alumnos respecto a la importancia de la comunicación efectiva como competencia transversal a nivel de máster, se ha diseñado un cuestionario para realizar una encuesta anónima. Con el objeto de obtener la información necesaria para realizar la investigación, el cuestionario se ha dividido en dos partes: la primera trata de caracterizar al individuo, preguntando el tipo de máster que está realizando, el sexo, la edad, la titulación de entrada al máster y la procedencia; en la segunda se plantearon 12 preguntas para conocer la opinión del encuestado respecto a la importancia de la comunicación efectiva utilizando una escala Likert de 5 opciones para las respuestas: 1) muy poco importante, 2) poco importante, 3) a medias, 4) importante, 5) muy importante. En el trabajo de Yepes (2014) se siguió una metodología similar.

\subsection{Caracterización de la encuesta realizada}

Se ha realizado una muestra de conveniencia no probabilística a los alumnos del MIUH y del MAPGIC. La recogida de datos se realizó en el segundo cuatrimestre del curso 2014- 
15. El tamaño de la muestra ha sido $N=43$, de los cuales 17 (39,5\%) lo han sido del MIUH y $26(60,5 \%)$ del MAPGIC. El nivel de confianza utilizado es del $95 \%$, con $p=q=0,5$, lo cual implica un error muestral del $15,2 \%$, suponiendo que la muestra representa una población infinita. Por otra parte, el análisis de fiabilidad medido a través del $\alpha$ de Cronbach, que es un indicador de la homogeneidad o consistencia interna de la escala de los ítems utilizados (P1 a P12), ha dado 0,882, lo cual se considera suficientemente alto. La herramienta de tratamiento de datos y análisis estadístico ha sido SPSS 17. A continuación se examinan las variables y se aplica un análisis multivariante para interpretar los resultados.

El perfil del encuestado corresponde con un alumno español del MAPGIC, con una edad entre 24 y 30 años, ingeniero civil o ITOP. En efecto, los alumnos encuestados fueron 26 (60,5\%), mientras que las alumnas fueron 17 (39,5\%). En cuanto a grupos de edades, entre 20 y 23 años respondieron 3 (7,0\%), entre 24 y 30 años respondieron 31 (72,1\%) y con 30 o más contestaron 9 (20,9\%). En cuanto a las titulaciones fueron ingeniero civil o ITOP (62,8\%), arquitecto técnico (15,3\%), arquitecto $(11,6 \%)$ e ingeniero de caminos $(7,0 \%)$. La procedencia de España (51,2\%) e Iberoamérica (41,9\%).

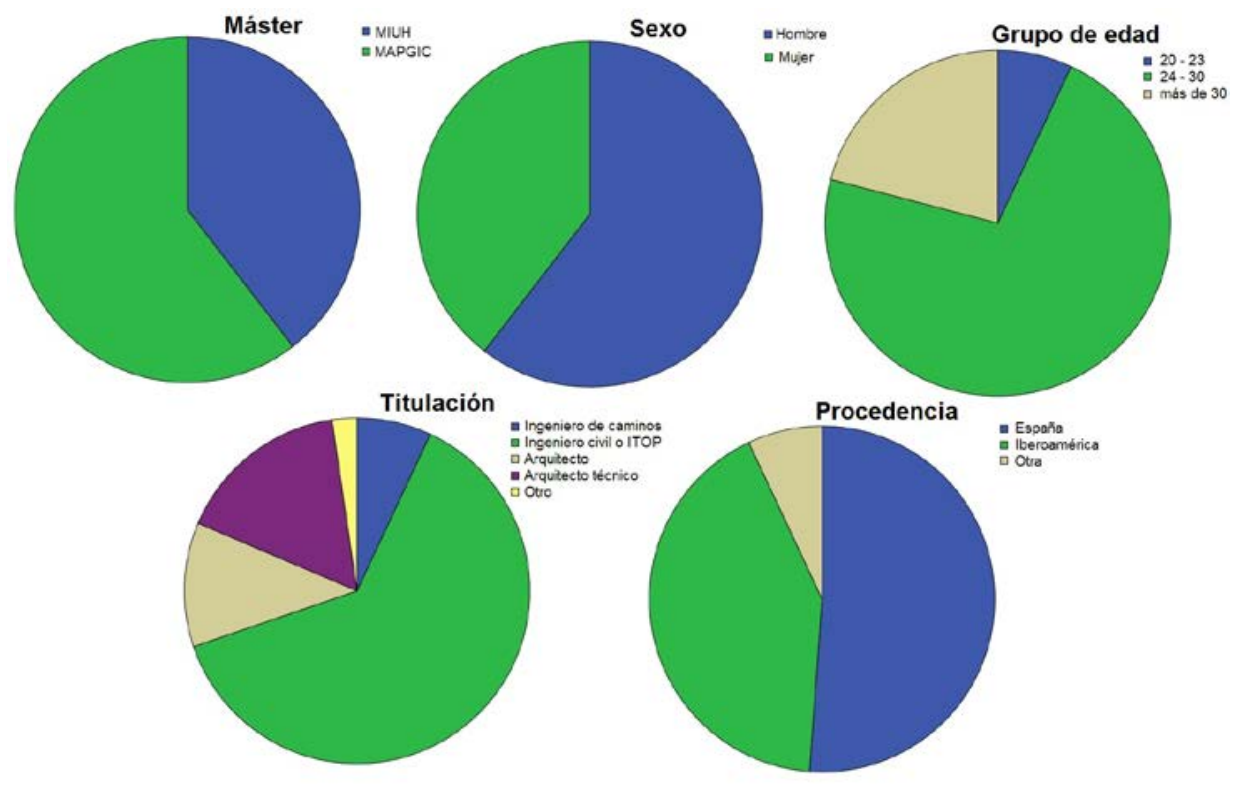

Fig. 2 Caracterización de la muestra analizada

\subsection{Análisis estadístico descriptivo}

La Tabla 1 recoge la media y la desviación típica obtenidas para cada una de las 12 preguntas realizadas a los encuestados. Se comprueba que, aquellos resultados de 
La competencia transversal de comunicación efectiva en estudios de máster en el ámbito de la ingeniería civil y la construcción

aprendizaje a los que se da mayor importancia y están más de acuerdo es (1) organizar y expresar correctamente las ideas y conocimientos en una presentación oral, (2) redactar textos y documentos con un contenido coherente, con un nivel básico de corrección ortográfica y gramatical y con la estructura y el estilo adecuados al tipo de público y los objetivos de la comunicación, y (3) comunicarse de forma clara y eficaz en una presentación oral, utilizando las estrategias y los medios adecuados, en función del público y los objetivos. Los dos primeros objetivos de aprendizaje corresponden a un nivel de grado, y sólo el tercero es de nivel de máster.

Tabla 1. Media y desviación típica de las respuestas al cuestionario

\begin{tabular}{|c|c|c|c|}
\hline $\mathbf{N}^{\mathbf{o}}$ & Pregunta & Media & D. Típ. \\
\hline P3 & $\begin{array}{l}\text { Organizar y expresar correctamente las ideas y conocimientos en una } \\
\text { presentación oral }\end{array}$ & 4,67 & ,606 \\
\hline P1 & $\begin{array}{l}\text { Redactar textos y documentos con un contenido coherente, con un nivel } \\
\text { básico de corrección ortográfica y gramatical y con la estructura y el } \\
\text { estilo adecuados al tipo de público y los objetivos de la comunicación }\end{array}$ & 4,58 & ,663 \\
\hline P8 & $\begin{array}{l}\text { Comunicarse de forma clara y eficaz en una presentación oral, utilizando } \\
\text { las estrategias y los medios adecuados, en función del público y de los } \\
\text { objetivos }\end{array}$ & 4,58 & ,698 \\
\hline P6 & Argumentar y justificar razonadamente la información que se expone & 4,51 & ,703 \\
\hline $\mathrm{P} 2$ & $\begin{array}{l}\text { Escuchar atentamente y responder adecuadamente cuando se le formulen } \\
\text { preguntas en una presentación oral }\end{array}$ & 4,44 & ,700 \\
\hline P11 & Saber comunicarse en inglés profesional en una comunicación oral & 4,44 & ,765 \\
\hline $\mathrm{P} 10$ & Saber escribir informes o artículos en un inglés profesional correcto & 4,40 & ,791 \\
\hline P12 & $\begin{array}{l}\text { Comprender, sin ayuda del diccionario, el contenido de un informe en } \\
\text { inglés }\end{array}$ & 4,33 & ,778 \\
\hline P5 & Exponer e interpretar resultados según diferentes públicos y objetivos & 4,28 & ,701 \\
\hline P9 & $\begin{array}{l}\text { Analizar, valorar y responder las preguntas que se le formulen en una } \\
\text { presentación oral, razonando las respuestas concienzudamente }\end{array}$ & 4,16 & ,721 \\
\hline P7 & $\begin{array}{l}\text { Elaborar gráficos profesionales efectivos para explicar, interpretar, } \\
\text { evaluar y argumentar información en función del público y los objetivos }\end{array}$ & 4,09 & ,781 \\
\hline P4 & $\begin{array}{l}\text { Redactar y revisar documentos con el formato, contenido, estructura, } \\
\text { corrección lingüística y registro adecuados, según el tipo de público y los } \\
\text { objetivos de la comunicación }\end{array}$ & 3,98 & ,740 \\
\hline
\end{tabular}


Por otra parte, los resultados de aprendizaje donde existen mayores discrepancias son (1) el saber escribir informes o artículos en un inglés profesional correcto, y (2) elaborar gráficos profesionales efectivos para explicar, interpretar, evaluar y argumentar la información en función del público y los objetivos. El resultado de aprendizaje al que se de la una menor importancia es redactar y revisar documentos con el formato, contenido, estructura, corrección lingüística y registro adecuados, según el tipo de público y los objetivos de la comunicación

Tras realizar un análisis de correlaciones entre las preguntas realizadas, la más fuerte (correlación de Pearson de 0,845, con significación bilateral al nivel 0,01) corresponde a la importancia del saber comunicarse en inglés profesional en una comunicación oral (P11) y saber escribir informes o artículos en un inglés profesional correcto P10). La siguiente correlación más fuerte (correlación de Pearson de 0,624, con una significación bilateral al nivel 0,01 ) es la relación entre organizar y expresar correctamente las ideas y conocimientos en una presentación oral (P3) con comunicarse de forma clara y eficar en una presentación oral, utilizando las estrategias y los medios adecuados (P8). Además, P3 se correlaciona fuertemente $(0,624$ y significación bilateral al nivel 0,01$)$ con argumentar y justificar razonadamente la información que se expone (P6).

Se ha comprobado, realizando la prueba no paramétrica de Welch, que existe una diferencia significativa ( $\mathrm{p}$-valor $=0,002$ ) entre la mayor importancia que le dan los alumnos del MAPGIC $(4,73)$ que los del MIUH $(3,88)$ a saber escribir informes o artículos en un inglés profesional correcto (P10). Lo mismo ocurre con P11, saber comunicarse en inglés profesional en una comunicación oral (MAPGIC, 4,77; MIUH, 3,94), con p-valor = 0,002. Esta diferente importancia frente al inglés también muestra diferencias significativas entre los españoles y los iberoamericanos. Así , para P10, españoles 4,18 e iberoaméricanos 4,78, con un p-valor $=0,03$ con la prueba de Welch.

\subsection{Aplicación del análisis multivariante}

A continuación se realiza un análisis factorial mediante el método de componentes principales (Yepes et al., 2009) para identificar las variables subyacentes o factores que expliquen la configuración de las correlaciones dentro del conjunto de variables observadas. En definitiva, se quiere averiguar los “constructos” o variables subyacentes que permitan explicar la mayoría de la varianza observada. Además, se va a realizar un análisis de regresión lineal múltiple para intentar explicar al máximo la valoración de "comunicarse de forma clara y eficaz en una presentación oral, utilizando las estrategias y los medios adecuados, en función del público y los objetivos”.

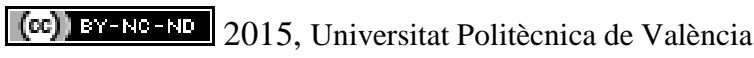

Congreso In-Red (2015) 
La competencia transversal de comunicación efectiva en estudios de máster en el ámbito de la ingeniería civil y la construcción

\subsubsection{Análisis de componentes principales}

El análisis de componentes principales examina la interdependencia entre variables para reducir la dimensión de un conjunto original de variables a un nuevo subconjunto formado por variables no observables. En síntesis, calcula unos factores que sean combinación lineal de las variables originales y que, además, sean independientes entre sí. La primera componente principal se escoge de forma que explique la mayor parte de la varianza posible de las variables originales, y así sucesivamente. Esta técnica no presupone una dependencia a priori entre las variables, y por tanto, se aplica antes de iniciar una regresión múltiple (Shaw, 2003). Para evitar que la unidad de medida influya en los resultados, se ha empleado la matriz de correlaciones en lugar de la de covarianzas. De este modo, el valor medio de los componentes principales es 0 y su desviación típica, 1 . Además, se ha tomado como criterio para determinar el número de componentes principales el que su autovalor sea superior a la unidad. Asimismo, para facilitar la interpretación, se ha empleado el método Varimax, que supone una rotación ortogonal que minimiza el número de variables que tienen saturaciones altas en cada factor (Kaiser, 1958).

Antes de realizar la extracción de los componentes principales, cada una de las variables queda explicada al $100 \%$ por ella misma. Sin embargo, una vez extraídas las componentes principales, éstas no explican toda la variabilidad de cada variable, pues se pierde información. En la Tabla 2 queda reflejada la desviacion estandarizada tras la extracción, es decir, las comunalidades, que miden el grado de información que tenemos tras dicha extracción. Lo que mejor explica el modelo es la pregunta 11 (saber comunicarse en inglés profesional en una comunicación oral), y la que menos la pregunta 1 (redactar textos y documentos con un contenido coherente, con un nivel básico de corrección ortográfica y gramatical y con la estructura y el estilo adecuados al tipo de público y los objetivos de la comunicación).

Con los criterios expuestos, subyacen 3 componentes principales que son capaces de explicar el 65, 9\% de la varianza de las 12 preguntas de la encuesta realizada (ver Tabla 3). Los componentes tienen que ver con los siguientes aspectos subyacentes:

- Componente 1: Competencias relacionadas con la comunicación oral

- Componente 2: Competencia relacionada con la organización y claridad de las ideas (responder preguntas y redactar correctamente)

- Componente 3: Competencia relacionada con el uso del idioma inglés

La Tabla 4 recoge la matriz factorial de los componentes rotados, que indica la correlación existente entre cada uno de los componentes principales y las variables originales. Lo que representa son los pesos de cada variable en la relación lineal de cada componente principal con las distintas variables. 
Tabla 2. Comunalidades

\begin{tabular}{|c|c|c|}
\hline $\mathbf{N}^{\mathbf{0}}$ & Pregunta & Extracción \\
\hline P11 & Saber comunicarse en inglés profesional en una comunicación oral & 910 \\
\hline $\mathrm{P} 10$ & Saber escribir informes o artículos en un inglés profesional correcto & ,813 \\
\hline P6 & Argumentar y justificar razonadamente la información que se expone & ,691 \\
\hline P7 & $\begin{array}{l}\text { Elaborar gráficos profesionales efectivos para explicar, interpretar, } \\
\text { evaluar y argumentar información en función del público y los objetivos }\end{array}$ & ,689 \\
\hline P3 & $\begin{array}{l}\text { Organizar y expresar correctamente las ideas y conocimientos en una } \\
\text { presentación oral }\end{array}$ & ,684 \\
\hline P5 & Exponer e interpretar resultados según diferentes públicos y objetivos & ,661 \\
\hline $\mathrm{P} 2$ & $\begin{array}{l}\text { Escuchar atentamente y responder adecuadamente cuando se le formulen } \\
\text { preguntas en una presentación oral }\end{array}$ & ,657 \\
\hline P9 & $\begin{array}{l}\text { Analizar, valorar y responder las preguntas que se le formulen en una } \\
\text { presentación oral, razonando las respuestas concienzudamente }\end{array}$ & ,654 \\
\hline P8 & $\begin{array}{l}\text { Comunicarse de forma clara y eficaz en una presentación oral, utilizando } \\
\text { las estrategias y los medios adecuados, en función del público y de los } \\
\text { objetivos }\end{array}$ &, 581 \\
\hline P12 & $\begin{array}{l}\text { Comprender, sin ayuda del diccionario, el contenido de un informe en } \\
\text { inglés }\end{array}$ &, 544 \\
\hline P4 & $\begin{array}{l}\text { Redactar y revisar documentos con el formato, contenido, estructura, } \\
\text { corrección lingüística y registro adecuados, según el tipo de público y los } \\
\text { objetivos de la comunicación }\end{array}$ &, 537 \\
\hline $\mathrm{P} 1$ & $\begin{array}{l}\text { Redactar textos y documentos con un contenido coherente, con un nivel } \\
\text { básico de corrección ortográfica y gramatical y con la estructura y el estilo } \\
\text { adecuados al tipo de público y los objetivos de la comunicación }\end{array}$ & 490 \\
\hline
\end{tabular}

Tabla 3. Varianza total explicada

\begin{tabular}{cccc}
\hline & \multicolumn{3}{c}{ Autovalores iniciales } \\
\hline Componente & Total & \% de la varianza & \% acumulado \\
\hline 1 & 5,318 & 44,319 & 44,319 \\
2 & 1,495 & 12,455 & 56,773 \\
3 & 1,099 & 9,161 & 65,934 \\
\hline
\end{tabular}


La competencia transversal de comunicación efectiva en estudios de máster en el ámbito de la ingeniería civil y la construcción

Tabla 4. Matriz de componentes rotados

\begin{tabular}{|c|c|c|c|c|}
\hline & Componentes & & & \\
\hline $\mathbf{N}^{0}$ & Pregunta & 1 & 2 & 3 \\
\hline P7 & $\begin{array}{l}\text { Elaborar gráficos profesionales efectivos para explicar, } \\
\text { interpretar, evaluar y argumentar información en función del } \\
\text { público y los objetivos }\end{array}$ & ,817 & & \\
\hline P8 & $\begin{array}{l}\text { Comunicarse de forma clara y eficaz en una presentación oral, } \\
\text { utilizando las estrategias y los medios adecuados, en función del } \\
\text { público y de los objetivos }\end{array}$ & ,702 & & \\
\hline P3 & $\begin{array}{l}\text { Organizar y expresar correctamente las ideas y conocimientos en } \\
\text { una presentación oral }\end{array}$ & ,684 & & \\
\hline P6 & $\begin{array}{l}\text { Argumentar y justificar razonadamente la información que se } \\
\text { expone }\end{array}$ & ,678 & & \\
\hline P5 & $\begin{array}{l}\text { Exponer e interpretar resultados según diferentes públicos y } \\
\text { objetivos }\end{array}$ & ,651 & & \\
\hline P2 & $\begin{array}{l}\text { Escuchar atentamente y responder adecuadamente cuando se le } \\
\text { formulen preguntas en una presentación oral }\end{array}$ & & ,769 & \\
\hline P9 & $\begin{array}{l}\text { Analizar, valorar y responder las preguntas que se le formulen en } \\
\text { una presentación oral, razonando las respuestas concienzudamente }\end{array}$ & & ,725 & \\
\hline P4 & $\begin{array}{l}\text { Redactar y revisar documentos con el formato, contenido, } \\
\text { estructura, corrección lingüística y registro adecuados, según el } \\
\text { tipo de público y los objetivos de la comunicación }\end{array}$ & & ,696 & \\
\hline $\mathrm{P} 1$ & $\begin{array}{l}\text { Redactar textos y documentos con un contenido coherente, con un } \\
\text { nivel básico de corrección ortográfica y gramatical y con la } \\
\text { estructura y el estido adecuados al tipo de público y los objetivos } \\
\text { de la comunicación }\end{array}$ & & ,532 & \\
\hline P11 & $\begin{array}{l}\text { Saber comunicarse en inglés profesional en una comunicación } \\
\text { oral }\end{array}$ & & & ,929 \\
\hline $\mathrm{P} 10$ & $\begin{array}{l}\text { Saber escribir informes o artículos en un inglés profesional } \\
\text { correcto }\end{array}$ & & & ,868 \\
\hline $\mathrm{P} 12$ & $\begin{array}{l}\text { Comprender, sin ayuda del diccionario, el contenido de un } \\
\text { informe en inglés }\end{array}$ & & & ,617 \\
\hline & $\begin{array}{l}\text { Método de extracción: Análisis de componentes principales. } \\
\text { Normalización Varimax con Kaiser. Se han suprimido valores absol }\end{array}$ & $\bar{M}$ & & $\begin{array}{l}\text { tacic } \\
5 .\end{array}$ \\
\hline
\end{tabular}




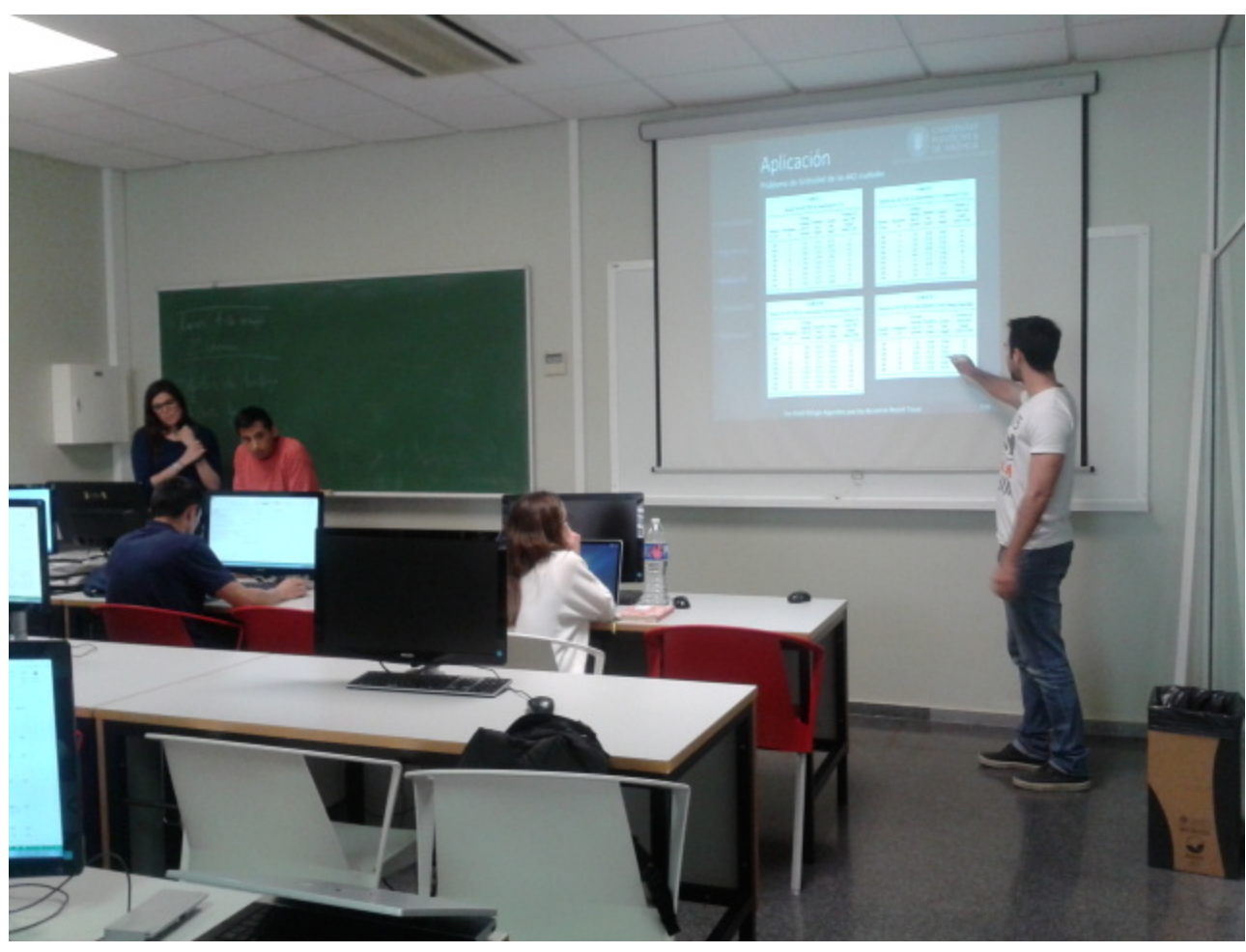

Fig. 1 Presentación oral de un artículo científico en la asignatura "Modelos predictivos y de optimización de estructuras de hormigón”, del MIUH.

\subsubsection{Modelos de regresión múltiple}

En este apartado realizamos un análisis de regresión de todas las variables para intentar establecer modelos que expliquen la variable dependiente que elijamos. Para ello se realizan inferencias acerca de modelos lineales simples o múltiples y se obtienen medidas cuantitativas del grado de relación de las variables a través del coeficiente de correlación $R$. Los modelos lineales se ajustan por mínimos cuadrados de forma que la variable dependiente se encuentre explicada lo máximo posible por un conjunto de variables independientes. La bondad del ajuste se evalúa mediante el coeficiente de determinación $R^{2}$, que se interpreta como la proporción de variación de la variable de respuesta explicada mediante el modelo de regresión lineal (Draper y Smith, 1999).

En primer lugar, se intenta explicar cada variable de respuesta en función de aquella variable independiente con la cual se encuentra más correlacionada. Se trata de aumentar el coeficiente de regresión incorporando variables independientes explicativas. Para ello se procede mediante el método stepwise de pasos sucesivos (Hocking, 1976), consistente en

\section{(cc)) EY-NC-ND 2015, Universitat Politècnica de València}

Congreso In-Red (2015) 
La competencia transversal de comunicación efectiva en estudios de máster en el ámbito de la ingeniería civil y la construcción

introducir las variables una por una y comprobar si la variable permanece o sale del modelo. Se toma como criterio de inclusión un incremento en la varianza explicada significativo al $5 \%(F=0,05)$, mientras que para excluir una variable se considera un decremento del $10 \%(F=0,10)$. La primera variable introducida es la que presenta un coeficiente de correlación $R$ más alto. A continuación se vuelven a calcular todas las correlaciones eliminando la influencia de aquella que ya ha entrado en el modelo, y se introduce la siguiente con mayor $R$; de esta forma se consigue que las variables que entren no sean dependientes de las que ya figuran en el modelo.

Como resultado de la regresión múltiple realizada (ver Tabla 5), se puede comprobar cómo comunicarse de forma clara y eficaz en una presentación oral se ver reforzado por el hecho de organizar y expresar correctamente las ideas y conocimientos y con la elaboración profesional de gráficos efectivos. Con estas dos variables, se puede explicar el 44,1\% de la variabilidad de la comunicación en una presentación oral. Es evidente que existen más factores que explican la variabilidad y no están recogidos en el modelo. Ello refuerza la utilidad del uso de rúbricas para evaluar la presentación oral.

Tabla 5. Modelos de regresión múltiple. Variable dependiente: P8 Comunicarse de forma clara y eficaz en una presentación oral, utilizando las estrategias y los medios adecuados, en función del público y los objetivos

\begin{tabular}{llcc}
\hline Modelo & Coef. & $\begin{array}{c}\boldsymbol{R}^{2} \\
\text { corregida }\end{array}$ \\
\hline 1 & $\begin{array}{l}\text { (Constante) } \\
\text { P3 Organizar y expresar correctamente las ideas y conocimientos en una } \\
\quad \text { presentación oral }\end{array}$ & 0,721 & 0,378 \\
\hline 2 & $\begin{array}{l}\text { (Constante) } \\
\text { P3 Organizar y expresar correctamente las ideas y conocimientos en una }\end{array}$ & 0,785 & \\
& $\begin{array}{l}\text { presentación oral } \\
\text { P7 Elaborar gráficos profesionales efectivos para explicar, interpretar, } \\
\text { evaluar y argumentar información en función del público y los objetivos }\end{array}$ & 0,269 & \\
\hline
\end{tabular}




\section{Conclusiones}

Se comprueba que, aquellos resultados de aprendizaje a los que se da mayor importancia y están más de acuerdo es (1) organizar y expresar correctamente las ideas y conocimientos en una presentación oral, (2) redactar textos y documentos con un contenido coherente, con un nivel básico de corrección ortográfica y gramatical y con la estructura y el estilo adecuados al tipo de público y los objetivos de la comunicación, y (3) comunicarse de forma clara y eficaz en una presentación oral, utilizando las estrategias y los medios adecuados, en función del público y los objetivos. Por otra parte, los resultados de aprendizaje donde existen mayores discrepancias son (1) el saber escribir informes o artículos en un inglés profesional correcto, y (2) elaborar gráficos profesionales efectivos para explicar, interpretar, evaluar y argumentar la información en función del público y los objetivos. El resultado de aprendizaje al que se de la una menor importancia es redactar y revisar documentos con el formato, contenido, estructura, corrección lingüística y registro adecuados, según el tipo de público y los objetivos de la comunicación.

Con los criterios expuestos, subyacen tres componentes principales que son capaces de explicar el 65, 9\% de la varianza de las 12 preguntas de la encuesta realizada. Los componentes tienen que ver con los siguientes aspectos subyacentes:

- Componente 1: Competencias relacionadas con la comunicación oral

- Componente 2: Competencia relacionada con la organización y claridad de las ideas (responder preguntas y redactar correctamente)

- Componente 3: Competencia relacionada con el uso del idioma inglés

A la vista de los resultados, se considera coherente la aplicación de las metodologías activas basadas en el estudio del caso y en el trabajo en grupo para realizar las siguientes actividades: presentación oral en grupo de un documento científico y redacción en formato científico de los resultados de un pequeño trabajo de investigación. Estas actividades servirán para evaluar los resultados de aprendizaje de la competencia transversal comunicación efectiva.

\section{Agradecimientos}

Los autores agradecen la colaboración de los alumnos del MUIH y del MAPGIC, así como el apoyo recibido por el Ministerio de Ciencia e Innovación (Proyecto de Investigación BIA2014-56574-R) y por la Universitat Politècnica de València (Equipo de Innovación y Calidad Educativa EXCELCON). 
La competencia transversal de comunicación efectiva en estudios de máster en el ámbito de la ingeniería civil y la construcción

\section{Referencias}

DRAPER, N.; SMITH, H. (1999). Applied regression analysis. New York: Wiley.

ENAEE. The EUR-ACE ${ }^{\circledR}$ System <http://www.enaee.eu/eur-ace-system> [Consulta: 7 de mayo de 2015]

HOCKING, R. (1976). “The analysis and selection of variables in linear regression” en Biometrics, vol. 32, p. 1-49.

KAISER, H.F. (1958). "The Varimax criterion for analytic rotation in factor analysis” en Psychometrika, vol. 23, issue 3, p. 187-200.

PELLICER, E.; YEPES, V.; ORTEGA, A.J. (2013). “Method for planning a graduate program in construction management”, en Journal of Professional Issues in Engineering Education and Practice ASCE, vol 139, issue 1,p. 33-41

PELLICER, E.; YEPES, V.; TEIXEIRA, J.C.; MOURA, H.P.; CATALÁ, J. (2014). Construction Management. New York: Wiley Blackwell.

SHAW, P.J.A. (2003). Multivariate statistics for the environmental science. London: Hoddeer-Arnold.

UNIVERSITAT POLITÈCNICA DE VALÉNCIA. Competencias transversales $<$ http://competencias.webs.upv.es/wp/> [Consulta: 7 de mayo de 2015]

UNIVERSITAT POLITÈCNICA DE VALÈNCIA. Excelcon. ¿Cómo podemos evaluar una presentación oral? <http://excelcon.blogs.upv.es/2015/02/06/como-podemos-evaluaruna-presentacion-oral/> [Consulta: 7 de mayo de 2015]

UNIVERSITAT POLITÈCNICA DE VALÈNCIA. Excelcon. ¿Tienen nuestros alumnos que aprender a escribir artículos científicos?

$<$ http://excelcon.blogs.upv.es/2014/03/10/tienen-nuestros-alumnos-que-aprender-aescribir-articulos-cientificos/> [Consulta: 7 de mayo de 2015]

UNIVERSITAT POLITÈCNICA DE VALÈNCIA. Excelcon. Estudio de caso. Lectura y comprensión de un artículo científico.

<http://excelcon.blogs.upv.es/2013/05/20/estudio-de-caso-lectura-y-comprension-deun-articulo-cientifico/> [Consulta: 7 de mayo de 2015]

YEPES, V. (2014). "El uso del blog y las redes sociales en la asignatura de Procedimientos de Construcción”. En: Jornadas de Innovación Educativa y Docencia en Red IN-RED 2014. 15-16 de julio, Valencia, pp. 1-9.

YEPES, V. ; DÍAZ, J., GONZÁLEZ-VIDOSA, F.; ALCALÁ, J. (2009). “Caracterización estadística de tableros pretensados para carreteras” en Revista de la Construcción, vol. 8, issue 2, p. 95-109.

YEPES, V.; PELLICER, E.; ORTEGA, J.A. (2012). "Designing a benchmark indicator for managerial competences in construction at the graduate level" en Journal of Professional Issues in Engineering Education and Practice ASCE, vol. 138, issue 1, p. 48-54. 